\title{
Software evolution: The lifetime of fine-grained elements
}

\author{
Diomidis Spinellis ${ }^{\text {Corresp., } 1,2}{ }^{,}$Panos Louridas ${ }^{1}$, Maria Kechagia ${ }^{3}$ \\ ${ }^{1}$ Department of Management Science and Technology, Athens University of Economics and Business, Athens, Greece \\ 2 Department of Software Technology, Delft University of Technology, Delft, The Netherlands \\ 3 Department of Computer Science, University College London, University of London, London, United Kingdom \\ Corresponding Author: Diomidis Spinellis \\ Email address: dds@aueb.gr
}

A model regarding the lifetime of individual source code lines or tokens can estimate maintenance effort, guide preventive maintenance, and, more broadly, identify factors that can improve the efficiency of software development. We present methods and tools that allow tracking of each line's or token's birth and death. Through them, we analyze 3.3 billion source code element lifetime events in 89 revision control repositories. Statistical analysis shows that code lines are durable, with a median lifespan of about 2.3 years, and that young lines are more likely to be modified or deleted, following a Weibull distribution with the associated hazard rate decreasing over time. This behavior appears to be independent from specific characteristics of lines or tokens, as we could not determine factors that influence significantly their longevity across projects. The programming language, and developer tenure and experience were not found to be significantly correlated with line or token longevity, while project size and project age showed only a slight correlation. 


\title{
Software evolution:
}

\section{The lifetime of fine-grained elements}

\author{
Diomidis Spinellis ${ }^{1,2}$, Panos Louridas ${ }^{1}$, and Maria Kechagia ${ }^{3}$

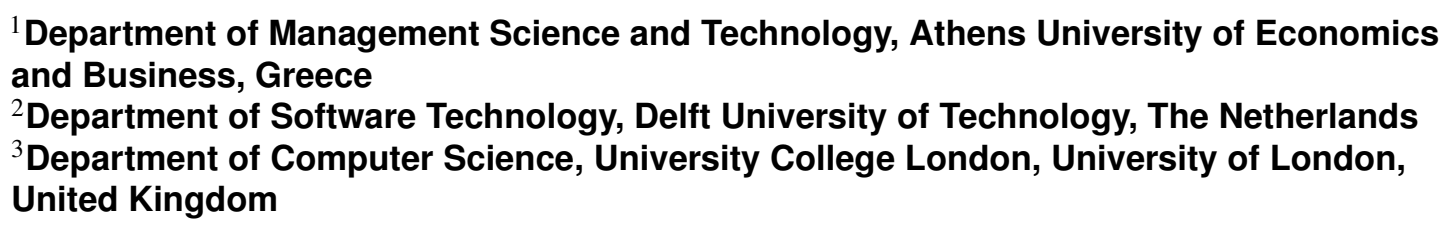

\begin{abstract}
A model regarding the lifetime of individual source code lines or tokens can estimate maintenance effort, guide preventive maintenance, and, more broadly, identify factors that can improve the efficiency of software development. We present methods and tools that allow tracking of each line's or token's birth and death. Through them, we analyze 3.3 billion source code element lifetime events in 89 revision control repositories. Statistical analysis shows that code lines are durable, with a median lifespan of about 2.3 years, and that young lines are more likely to be modified or deleted, following a Weibull distribution with the associated hazard rate decreasing over time. This behavior appears to be independent from specific characteristics of lines or tokens, as we could not determine factors that influence significantly their longevity across projects. The programming language, and developer tenure and experience were not found to be significantly correlated with line or token longevity, while project size and project age showed only a slight correlation.
\end{abstract}

\section{INTRODUCTION}

Although there is a significant body of work regarding the macroscopic characteristics (González-Barahona et al., 2009) and even laws (Lehman, 1980) of software evolution (Herraiz et al., 2013), much less is known about how software evolves at the microscopic scale, namely at the level of lines, statements, expressions, and individual tokens. A study of such details, apart from its self-supporting merits as curiosity-driven empirical research, can derive results that can in the future be used for improving software development processes (Humphrey, 1989, p. 3), architecting software systems (Barnes et al., 2013; Breivold et al., 2012), developing machine learning algorithms (Allamanis et al., 2018; Alon et al., 2019), organizing software development teams (Rodríguez et al., 2012), estimating maintenance effort (Albrecht and Gaffney, 1983; Atkins et al., 2002; Zimmermann et al., 2005), designing new features for configuration management systems (Jiang et al., 2017; White et al., 2015), locating software faults (Cotroneo et al., 2013; Giger et al., 2011; Kechagia et al., 2019; Salfner et al., 2010), guiding probabilistic programming (Gordon et al., 2014), and enhancing programming languages (Vallée-Rai et al., 2010). Here we report on methods, tools, and the results we obtained by studying the lifetime of unmodified code lines and tokens in 89 revision control repositories over 3.3 billion source code element lifetime events.

At the point where the rubber hits the road, software consists of code lines. Their number has been extensively studied to gain insights on topics ranging from development effort (Albrecht and Gaffney, 1983; Gousios et al., 2008; Lind and Vairavan, 1989) and quality (Buse and Weimer, 2008; Kan, 2002; Stamelos et al., 2002; Zhang, 2009) to software growth (van Genuchten and Hatton, 2013; Godfrey and Tu, 2000; Hatton et al., 2017; Herraiz et al., 2007). This work contributes to the study of software evolution by looking quantitatively, not at changes in the number of code lines, but how and why individual lines or tokens change over the software's lifetime.

First, consider how long a line of code survives in its initial form. As software evolves over time, 
some lines are added, others are deleted, and existing ones are modified. From the time that a line enters the code base of a project, for how long does it live, i.e., for how long does it remain there unchanged? Are lines of code more of a durable asset that will be around for the long time, or are they more like perishable assets, that will only remain for a short time? How is their lifetime related to factors such as a system's size or the employed programming language?

A process model of aging can be further elaborated through quantitative characteristics. These include the mathematical function that determines when existing lines are likely to "die". We define as the line's death its deletion or the modification of its non-whitespace elements, and further examine the validity of this construct by also looking at the lifetimes of individual tokens. In functions that are used to characterize decay processes, their characteristic unit is often expressed through the measure of median lifespan: $t_{1 / 2}$. If a line $i$ is added at time $t_{i, 1}$ and is changed or disappears at time $t_{i, 2}$, its lifespan is $t_{i, 2}-t_{i, 1}$. The median lifespan, over all lines of a project, is the median value of all line lifespans, that is, the median of $t_{i, 2}-t_{i, 1}$ for all $i$.

Now take an added line of code. When will this code be changed or be entirely removed and how does its age factor into this question? One can imagine three possibilities. The first, a high infant mortality scenario, in which new lines of code are often changed as developers fix newly-introduced faults and refactor the code. The second, a senescence scenario, has code lines become outdated and less useful as they age and therefore face increasing chances of being replaced. The third, stochastic scenario, has lines getting replaced mostly due to other factors through what appears to be a random process with regard to their age. In practice, it is likely that all three scenarios play a role, but it is still possible that one of them dominates the aging process.

Finally, consider some reasons for which a line may change. These include misunderstood requirements, a fault in the code, or changes cascading from other work. While these are typically qualitatively analyzed, one can also examine the factors associated with them, such as the line's complexity, its developer's seniority, the project's size or the employed programming language.

Apart from its theoretical importance, a model of code aging at the level of code lines is useful in several ways. Many potential applications are listed in the opening paragraph; here are two concrete examples. First, the model can inform managers where to direct maintenance effort, for example to reduce the acquired technical debt (Kruchten et al., 2012) or address newly-discovered security vulnerabilities (Ozment and Schechter, 2006; Penta et al., 2009; Shin et al., 2010). Under the infant mortality scenario old lines are likely to remain in a project for ages, so they should periodically receive some love and care to keep them up to date with modern practices. In contrast, under the senescence scenario these will gradually fade away, so effort invested in maintaining them may be wasted. Second, the function expressing code age and its coefficients for a specific project can be used to guide maintenance effort estimation. This is important because humans are often biased when estimating development effort (Løhre and Jørgensen, 2016). Simplistically, effort is often measured in terms of code lines (Albrecht and Gaffney, 1983; Gousios et al., 2008; Lind and Vairavan, 1989). Therefore, if, given the age of existing lines, we can estimate how many of the project's lines are likely to change in the next year, this, together with the project's estimated code growth rate (Hatton et al., 2017), can roughly determine the required development effort. More broadly and importantly, given that code lines require effort to change, identifying and controlling factors that promote longer-living lines - for instance through better abstraction mechanisms - can direct improvements in software development efficiency.

The contributions of this paper are the development of an efficient method and tools that allow the tracking of the birth and death of individual source code lines and tokens over periods that can span decades and the empirical analysis of 3.3 billion source code element lifetime events to answer the following research questions.

RQ1 For how long does a line of code or token live? The answer to this question determines whether code elements are durable or perishable.

RQ2 How is a line's or token's age related to the probability of its change or deletion? The answer tells us whether younger code elements are more vulnerable to change and deletion (infant mortality), or whether older ones are more frail (senescence), or whether there are no age-related hazards.

RQ3 What other product or process factors may influence a line's or a token's survival? We investigate this question along the following dimensions. 
RQ3a The line's characteristics, which may reveal change-prone programming constructs or drivers of change.

RQ3c The committer's experience and tenure; one might expect senior developers to write more stable code.

RQ3d The project's size, which might lend it inertia against change.

RQ3e The employed programming language, demonstrating whether some programming languages lend themselves for writing more stable (or, alternatively, flexible) code.

\section{METHODS}

We studied code aging at the level of individual source code lines by selecting a number of suitable revision control repositories to study, coming up with a way to handle merges of development branches, constructing a tool that can track the lifetime of individual lines across successive software releases, creating a process and tools to also study the lifetime of individual tokens, choosing the statistical methods that best suited the domain, and applying them to the collected data.

As recommended by Ince et al. (2012), the source code and data associated with our results are openly available online. $^{1}$

\section{Material Selection}

We ran our study on open source software repositories due to their liberal availability and the fact that this simplifies the replication of our findings. We selected the revision control repositories to study based on five objective criteria consistent with our research goals.

GitHub hosting We only selected projects whose history is available on GitHub. This decision simplified the methods we used to select the projects and to traverse a project's revisions. The choice to use only GitHub-hosted repositories is not as restrictive as it sounds, because nowadays even projects that use other version control systems and hosting often make a (typically read-only) Git version of their repository available on GitHub.

Longevity We selected projects with at least ten years of commit data in order to obtain enough samples for statistical analysis.

Active development The code in the repository had to be actively developed as determined by code commits. Obviously, code in dormant projects doesn't exhibit aging processes and cannot be usefully modelled. To examine projects that are actively developed we calculated the number of weeks over the project's lifetime in which at least one commit had occurred. We then selected projects in which commits occurred in at least $85 \%$ of the weeks to take into account vacation time. We examined activity at weekly granularity, because some open source developers may only work over weekends.

Popularity We selected projects having at least 100 GitHub "stars". Results from popular projects are likely to be relevant to more people than those from obscure ones. Studying code aging in small test projects or student exercises is less likely to yield results of industrial relevance.

Programming language To study source code evolution at the level of individual tokens as well as lines, we only selected projects whose main programming language, as reported in GHtorrent (Gousios and Spinellis, 2012), is supported by the tokenizer we used (Spinellis, 2019). These languages were selected based on their popularity among the projects selected using the other criteria, and cover $76 \%$ of the repositories initially selected. The languages processed are C, C\#, Java, PHP, and Python.

We performed the project selection through analytical processing of the GHtorrent data set (January 2018 version) based on the process described by Gousios and Spinellis (2017). The code information was obtained by downloading and processing each selected revision of the corresponding repository. Other than the stated selection criteria, we did not perform any other manual adjustments to add popular projects or exclude obscure ones. We ensured that our data set did not include duplicated projects by pairing it with a dataset for GitHub repository deduplication (Spinellis et al., 2020). From the one duplicate and two triplicate sets we thus found we retained the repositories with the longer commit history. Specifically, we chose github.com/drools jbpm/drools over kiegroup/drools and droolsjbpm/guvnor, lede-project/source over openwrt-mirror/openwrt and

\footnotetext{
${ }^{1}$ Data: https://doi.org/10.5281/zenodo.4319986 (3.5GB compressed, 69GB uncompressed); source code:
} https://doi.org/10.5281/zenodo.4319993. 
Table 1. Descriptive Statistics of the Analyzed Repositories and Aggregate Totals

\begin{tabular}{lrrrrrr}
\hline Metric & Min & Max & Median & Mean & $\sigma$ & Total \\
\hline All Files & 384 & 110737 & 2786 & 7986 & 17522 & 710798 \\
Analyzed Source Code Files & 165 & 73655 & 1291 & 4175 & 11177 & 371566 \\
Analyzed Source Code Lines (thousands) & 3 & 15699 & 298 & 935 & 2083 & 83204 \\
Analyzed Source Code Tokens (thousands) & 0 & 72480 & 1423 & 4537 & 9793 & 403804 \\
Committers & 29 & 2211 & 247 & 397 & 407 & 35374 \\
GHTorrent Project Duration (Years) & 11 & 29 & 12 & 14 & 4 & \\
Analyzed Branch Duration (Years) & 6 & 41 & 13 & 15 & 5 & \\
All Commits (thousands) & 6 & 299 & 19 & 39 & 51 & 3508 \\
Analyzed Commits (thousands) & 0 & 192 & 11 & 24 & 36 & 2150 \\
Line deaths (thousands) & 1 & 28680 & 827 & 2326 & 4482 & 207022 \\
Token deaths (thousands) & 0 & 157530 & 3266 & 9954 & 21516 & 885935 \\
Project Stars & 117 & 34193 & 878 & 2207 & 4289 & \\
Commit Density (weekly \%) & 85 & 100 & 93 & 93 & 5 & \\
\hline
\end{tabular}

openwrt/packages, and doctrine/doctrine2 over doctrine/dbal. In total we analyzed 89 projects, comprising at the end of the studied period 372 thousands of source code files, 83 millions of source code lines, 404 millions of source code tokens, and 2.2 millions of examined commits. In terms of source code lines, we analyzed 497 million code line lifetime events: the appearance of 290 and the demise of 207 millions of source code lines. In terms of individual source code tokens, we analyzed 2.2 billion code token lifetime events: the appearance of 1290 and the demise of 886 millions of source code tokens. Key metrics of the projects we analyzed are listed in Table 1.

\section{History Simplification}

Software development with distributed version control repositories is characterized by the frequent generation of feature development branches and their subsequent merging into a more mainstream trunk (German et al., 2016). For example, the repositories we analyzed contained a total of 243 thousand merges, or about three thousand per project. As we detail below, merges confuse and complicate the processing of history and therefore required devising a scheme to deal with them.

The confusion arises from the fact that a topological ordering implied by the directed acyclic graph structure of a project's commit history will lose code changes or their time ordering. (A topological ordering of a directed graph is a linear ordering of its vertices such that for every directed edge $a b$ from vertex $a$ to vertex $b$, the linear ordering has $a$ appear before $b$.) Applying the typically-used three-way merge algorithm will result in the loss of code modifications, because the common ancestor will no longer be correctly represented.

The complexity of merges has to do with how changes listed at the point of a merge can be automatically processed to obtain the code after it. In experiments we performed with diverse Git output options we found that the output of git log and also git format-patch was not a reliable way to reconstruct the state of a project from its history (Spinellis, 2016a). Consequently, the output could also not be used to track the lifetime of individual lines. Although we did not look for the root cause of these problems in depth, we only encountered them when working with merges, which leads us to believe that they were indeed caused by merges. Using Git's combined diff format for merges is also unlikely to help, because, according to Git's git diff documentation "Combined diff format was created for review of merge commit changes, and was not meant for apply". And if dealing with binary merges was not bad enough, handling $n$-way merges, such as those handled by Git's octopus-merge algorithm, added even more complexity to the problem.

Consequently, we decided to simplify the commit history into a linear ordering by removing the least essential branches and merges. The rationale behind this decision is that each merge point captures changes that have happened on both branches; if the time difference from the branch to the subsequent merge is not too large, then the represented lifetime of the affected lines does not change substantially. (See analysis in the threats to validity Section.) An additional advantage of this approach is that it presents 


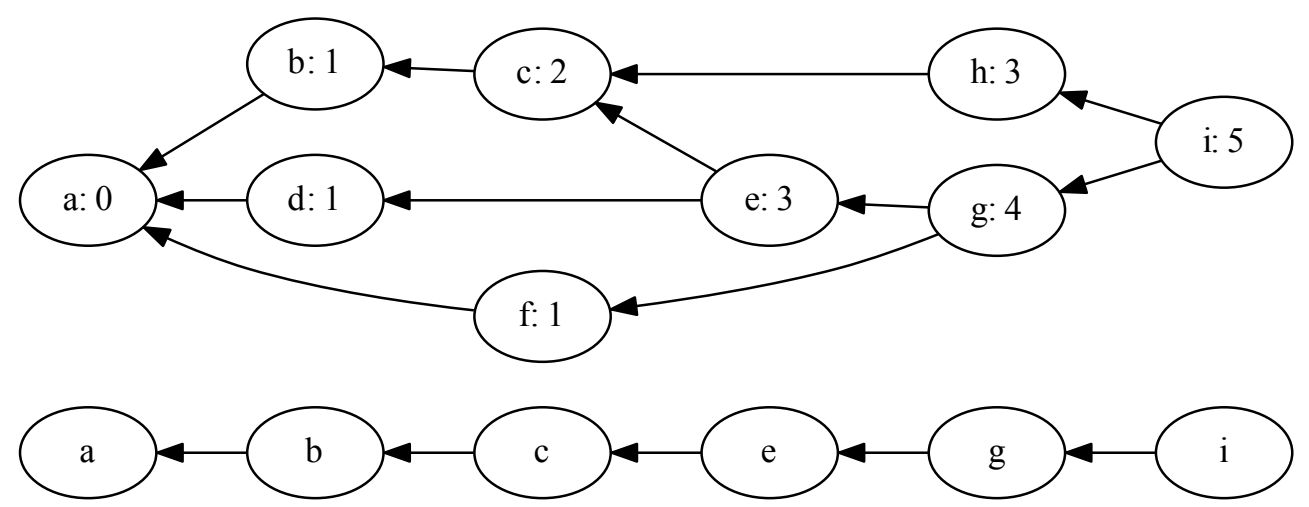

Figure 1. Branch graph path length attributes (top) and the longest path (bottom)

a commit sequence that is both topologically and temporarily ordered.

To obtain this linear ordering, we took the topological ordering of the project's commit graph and obtained the longest path in it. For directed acyclic graphs this path can be calculated in linear time, by having each vertex record its length as the maximum length of its parent neighbours plus one. Then the longest path can be obtained by traversing the graph along the vertices with the maximum recorded values. Figure 1 illustrates an example of a commit graph and its longest path. The simplification of history resulted in the reduction of examined commits from 3.106 million to 2.256 million, meaning that we processed about $73 \%$ of all commits.

\section{Lifetime Tracking}

One could in theory obtain an indication regarding the lifetime of individual lines by sampling the output of the Git's blame command at various time points. However, this process is computationally intensive and will only provide an approximation. To address these issues we designed an online algorithm and a corresponding open source tool (named lifetime) that continuously tracks the lifetime of code lines across successive commits.

Tracking the lifetime of individual code lines across code commits is not trivial. An earlier study that demonstrated the estimation of code decay in the Unix operating system source code over the period 1975-2015, employed the git blame command to record the age of lines at the point of each one of 71 releases (Spinellis, 2016b). Changes over the sampled releases in the cardinality of sets representing lines born at a specific point of time were then used to estimate the lifetime of the corresponding lines. However, this method is quite inaccurate, since the lifetime estimates are bound between the dates of two successive releases. Furthermore, it is also computationally expensive. The specific task required (on a massively parallel supercomputer) 9.9 core years CPU time, 3,815 cores, 7.6 TB RAM, and $588 \mathrm{~GB}$ of disk space. In fact, our case of $711 \mathrm{~K}$ files $\times 3.5 \mathrm{M}$ commits would require two orders of magnitude more resources.

In common with most version control systems, Git can output the differences in a file between two commits as a series of line additions and removals. (Changes are represented as an addition and removal.) By default, this operation uses the popular Myers algorithm (Myers, 1986) to minimize the presented differences. In common with the work by Zimmermann et al. (2006), we processed the output of Unix (Git) diff, rather than alternatives such as LHDiff (Asaduzzaman et al., 2013a), because diff operates fast and its output is machine-readable.

A line may appear in the output of a commit's differences for several reasons: 1) actual deletion within a retained file or through a file's deletion, 2) changes in identifier names, 3) other non-whitespace changes, 4) movement to another part of the same file, 5) movement to another file, 6) change of indentation, or 7) other cosmetic - whitespace - changes. Reasons 1 and 3 are definitely signs of the line's death that are relevant to this study: a (most-probably) functional modification. Our methods also consider as a line death reasons 2 and 4, because it is difficult to identify such changes with the tools we employed. We deal with these potential shortcomings by expanding our methods to track changes of individual tokens and by measuring the effect of line moves. We were also able to continue tracking through their lifetime lines that change due to reasons 5-7. First, we set git diff to ignore all changes in a line's whitespace. This filtered out noise introduced by indentation adjustments induced through 


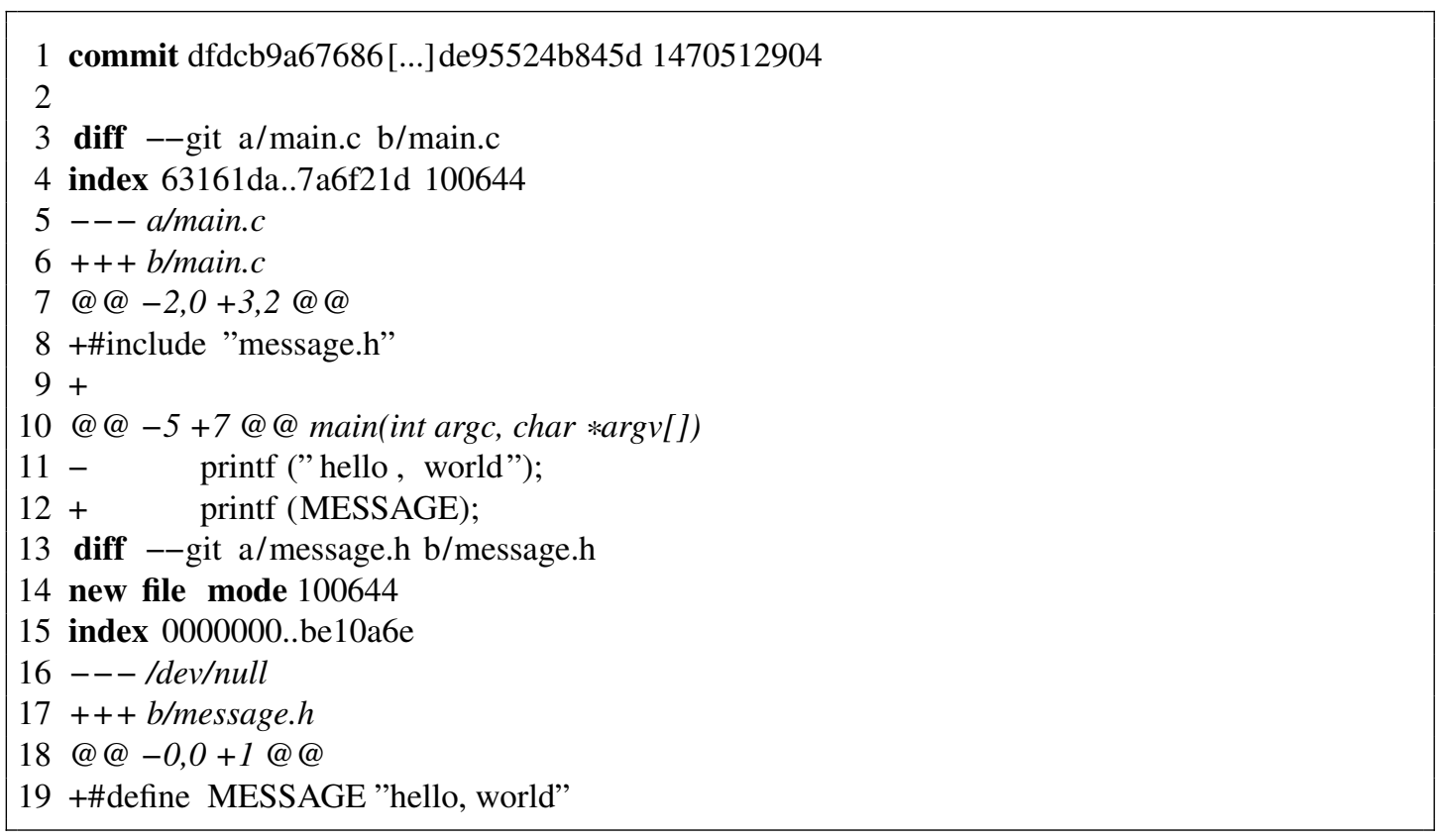

Listing 1. Example of git diff output

other changes as well as changes in a file's tab (hard Vs. soft tabs) or end-of-line (the use of line feed and carriage return) representation. Second, we configured git diff to detect file renaming and copying, in order to follow cross-file code movements.

An example of the git diff output format processed by the lifetime tool we built appears in Listing 1. Line 1 is a custom commit header we employed, containing the commit's SHA identifier and its timestamp. The commit involves changes to two files; lines 3 and 13 show the old and new names of the files being compared. When a file is removed or newly added the new file name (in the case of removals) or old file name (for additions) is / dev/null (the Unix empty file). Lines 4-6 and 15-17 contain metadata that is not important for the task at hand. Lines 7-9 show the addition of two lines at the new file range $+3,2$ listed in the @ @ line. Lines 18-19 do the same for the second (newly-created) file. Lines 10-12 show a line's change: line 5 of the old version's file is replaced by line 7 in the new version's file. in the new file version. A series of extended headers can appear after the diff line to indicate file deletions, renames, and copies, which Git detects by applying heuristics in the compressed repository state snapshot stored for each commit.

The lifetime program ${ }^{2}$ works by processing a series of git diff outputs (such as those detailed in the preceding paragraph) using the state machine depicted in Figure 2. State transitions take place when input lines match the regular expressions shown in the diagram.

The operations can be expressed through the following notation.

- The timestamp $T_{c}$ associated with commit currently being processed.

- A partial function $T:(F, L) \rightarrow T_{B}$ mapping each integer-numbered line $L$ of file $F$ onto its birth time $T_{B}$.

- Another partial function $B: F \rightarrow v$, that yields true when a file $F$ contains binary data (e.g. an image).

- The last line of each file $F_{E}=\max (\{l: T(F, l) \neq \perp\})$.

The rules applied when processing the git diff data are the following.

1. For each code line numbered $L$ added to file $F$ that the program encounters (e.g. lines 8, 9, 12, 19 in Listing 1), it remaps existing timestamps from $L$ until the end of the file $E$ in the map $T$ it maintains to make space for the new line, and it inserts an entry in the timestamp map with the current timestamp $T_{c}$

${ }^{2}$ Available in this study's source code package at https://doi.org/10.5281/zenodo. 4319993 and also on GitHub https://github.com/dspinellis/code-lifetime. 


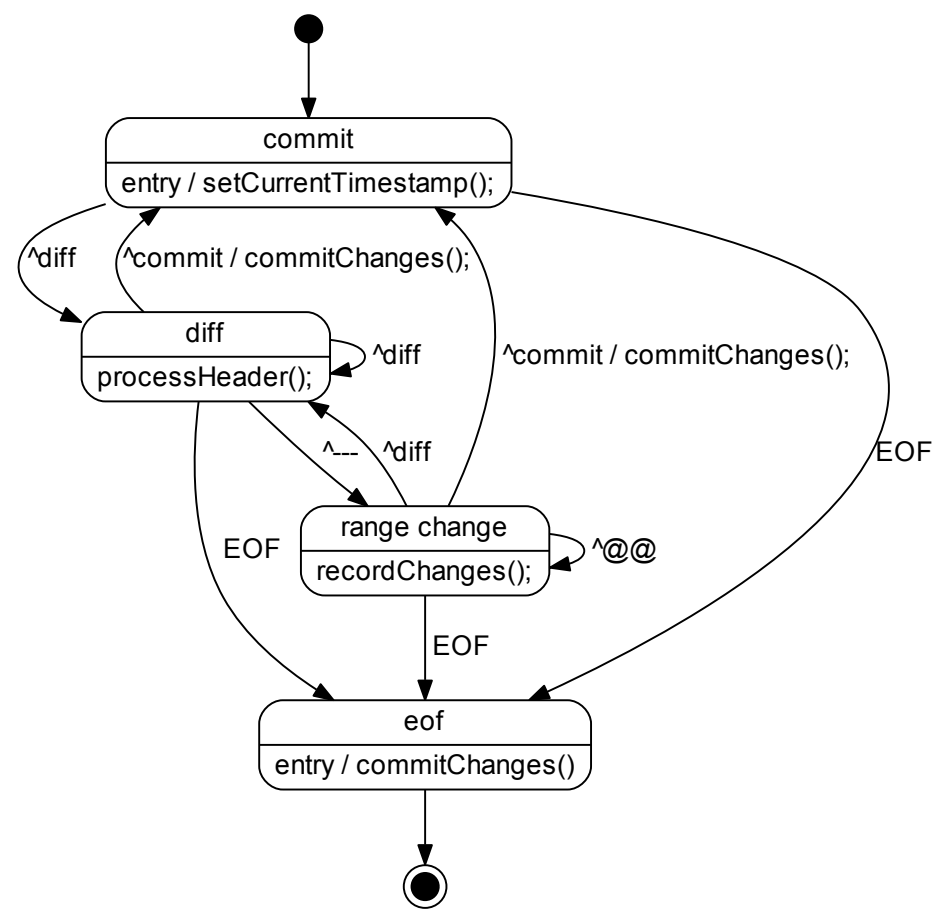

Figure 2. State machine for git diff output processing

(1470512904 in line 1 of Listing 1).

$$
\begin{aligned}
\forall l \in\left(L . . F_{E}\right), T^{\prime}(F, l+1) & =T(F, l) \\
T^{\prime}(F, L) & =T_{c}
\end{aligned}
$$

2. For each code line numbered $L$ deleted from file $F$ that the program encounters (e.g. line 11 in Listing 1), it outputs a tuple with the line's birth time and the time of its demise,

$$
\left(T(F, L), T_{c}\right)
$$

and it remaps the timestamps of the lines from $L$ until the end of the file $E$ to close-up the gap of the deleted line.

$$
\begin{aligned}
\forall l \in\left(L . . F_{E}-1\right), T^{\prime}(F, l) & =T(F, l+1) \\
T^{\prime}\left(F, F_{E}\right) & =\perp
\end{aligned}
$$

3. When changes to a binary file $F$ are encountered, this file is marked as binary in a map $B$, and no further output is ever performed on operations on that file. This is needed because changes to binary files are not identified in terms of lines, so further changes when a file reverts to text format will not have correct timestamps to refer to.

$$
B(F)=\text { true }
$$

4. When a file $F_{a}$ is identified as copied to file $F_{b}$, new map entries are established with the original line birth dates and the binary file status is also transferred to the new file.

$$
\begin{aligned}
\forall l \in\left(1 . . \max \left(F_{a E}, F_{b E}\right)\right), T\left(F_{b}, l\right) & =T\left(F_{a}, l\right) \\
B\left(F_{b}\right) & =B\left(F_{a}\right)
\end{aligned}
$$

5. When a file $F_{a}$ is identified as renamed to file $F_{b}$, new map entries are created as above, and the existing ones are removed.

6. After processing all commits, lifetime outputs tuples with the birth timestamps of all lines that are still alive and the word alive.

$$
\{(T(F, L), \text { alive }): T(F, L) \neq \perp\}
$$


The processing is complicated by the fact that all change references to the existing state of a commit, refer to the state before any change has been applied to any of the files; changes are not supposed to be applied while processing a commit. For example, if a commit renames file $F_{a}$ to $F_{b}$ and $F_{b}$ to $F_{a}$ the names of the two files will be correctly swapped. Also, changes to a file that has been copied or renamed in the same commit refer to the name of the file before the corresponding operation. This complication is addressed by recording all changes in a queue as instructions to add or remove elements from the timestamp map $T$. When all elements of a commit have been processed, a routine replays the recorded changes on the current state to generate the new one.

Considerable effort was invested in making the lifetime program easy to test and troubleshoot. This was needed for three reasons. First, the output of git-diff seems to be only informally defined and involves many special cases. Second, tracking line timestamps by hand to verify the program's operation is a complex and error prone process. Third, errors were encountered in the middle of processing data hundreds of gigabytes in size; isolating these errors proved to be a challenging task.

In order to help testing and troubleshooting the lifetime program supports eight command-line options that configure it to output diverse data regarding the processing it performs. A separate option can be used to terminate the processing at a specific commit, thus allowing the examination of the data at the given time point. The most important of the debug options, modifies the program's operation to store in the map $T$ the complete content of each added line, rather than the current timestamp $T_{c}$. Then, when a line is removed, it is compared with the map's content to verify that the two match. Any differences signify a failure in the process to record the changes. Such differences allowed us to find that the output of git log and git format-patch were not trustworthy enough for our purposes. Furthermore, the same debug option uses the map's contents to reconstruct a copy of the project's file tree, when all its commits have been processed. Comparing the file tree against a checked-out version of the project allows the end-to-end verification of the program's operation. The lifetime program is accompanied by two Git repositories containing tens of diverse commit cases. A test script is used to compare the reconstructed state against the original one at the end of each commit.

\section{Analysis of Individual Tokens}

Although lines of code are often used to measure software and its evolution, tracking changes at the level of lines can threaten the results' validity. Specifically, small changes, such as renaming an identifier, will appear to change many lines. In addition, a line may appear to change through edits unrelated to it, such as the addition of a brace when a statement is added below it. Consequently, it would be valuable to track evolution at the level of individual tokens rather than lines.

We designed and implemented a process and tools to track the birth and demise of individual tokens based on an idea by German et al. (2019). This involves creating a synthetic Git repository where files are stored as one token per line. The setup can be traced to the more general concept of using a synthetic Git repository to track arbitrary fine-grained elements (Hata et al., 2011). The downloaded repositories amount to $30 \mathrm{~GB}$ and the synthetic ones to $32 \mathrm{~GB}$. Tracking changes between revisions in such a repository will show the addition and removal of individual tokens, rather than complete lines. All other workflow and tools can remain the same.

We created tokenized versions of the selected repositories through two tools: a file tokenizer and a repository tokenizer. The repository tokenizer is a Perl script that acts as a filter between a git fast-export and a git fast-import command. It reads the dump of the original repository generated by the git fast-export command, queueing file content blobs it encounters, while passing the remaining data unchanged to its output. When it reads a commit packet, it matches the file extensions of the committed files against previously encountered blobs. For any blob whose file extension matches the languages supported by the file tokenizer, the repository tokenizer invokes the file tokenizer to convert the file into tokens, dumping the tokenized results on its output as the corresponding blob.

To tokenize the contents of each file, we used the tokenizer tool, which splits its input into tokens using simple look-ahead lexical analysis (Spinellis, 2019). Support for each language is provided through a separate lexical analyzer to cater for differences in operators, reserved words, commenting, and string delimiters. Through command-line options we directed the file tokenizer to split its input into a token per line replacing the content of strings and comments with an ellipsis (...), thus also allowing us to ignore non-code changes. 


\section{Analysis of Moved Lines}

Given that the file differencing program we employed will not report line moves within the same file, we attempted to quantify the effect of this behavior on our results. For this we developed a tool that uses Heckel's linear time differencing algorithm (Heckel, 1978), which does attempt to locate line moves. Although the output of this program is not suitable for running the fully fledged analysis, its summary of added and removed lines can be tallied against that of the git diff program to compare their performance in detecting lines that have not changed. By configuring git diff to run the alternative program between all successive revisions, we found that Heckel's algorithm, despite taking into account line moves, reports $2.2 \%$ more line additions and deletions than Git's stock algorithm. While differencing algorithms can always be tweaked to handle elaborate special cases, this result indicates that the differencing program we employed for the study works pretty hard to identify a competitively small set of differences, and that taking into account line moves using Heckel's algorithm would reduce the accuracy of our results by failing to track about $2 \%$ more lines.

\section{Effect of the Histogram Algorithm}

Following the recommendation of a recent systematic survey of studies that use diff (Nugroho et al., 2020), we also examined whether the use of Git's Histogram difference algorithm would substantially alter our results. Using a process similar to that described in the previous section, we measured the differences in the reported added and deleted lines between the Myers and the Histogram algorithms. Both differences were below $0.5 \%: 0.28 \%$ for deletions and $-0.45 \%$ for additions. The effect's small size is not surprising, because the Histogram algorithm mainly improves the readability of the provided patch.

\section{Statistical Analysis}

If we had the time of birth and the time of death for each line of code and token in a project we could estimate the median lifespan of the line or token directly, by calculating lifespans and finding their median value. We are interested in the median and not the mean, because lifetimes may be skewed, so the mean, or average, would not give a representative metric.

Unfortunately, we do not have the lifespans of all lines and tokens that we have tracked in the repositories we have examined. We do have their birth timestamps, but there are many lines and tokens that are still alive at the end of our follow-up period: these are the lines and the tokens that are part of the code base of a project at the last time we check. Their lifespans are right censored; they extend to the future. Across projects, the mean of the percentage of right censored lines is $29.97 \%$ and the median is $28.25 \%$; for tokens, the corresponding values are $33.38 \%$ and $30.87 \%$ respectively.

To estimate the median lifespan under such circumstances we use the Kaplan-Meier or product-limit survival estimate (E. L. Kaplan, 1958). If our measurements take place at times $t_{1}<t_{2}<\ldots<t_{n}$ and at time $t_{i}$ we have $n_{i}$ that are alive, of which $d_{i}$ die right at that moment, then the probability of being alive at time $t_{i}$ is given by:

$$
S\left(t_{i}\right)=S\left(t_{i-1}\right)\left(\frac{n_{i}-d_{i}}{n_{i}}\right)
$$

The recursive definition assumes $t_{0}<t_{1}$ and $S\left(t_{0}\right)=1$.

In our data, lines and tokens are born at different times during a project. Since we are interested in their lifespans and not at the chronological times of birth and death, we work only with the differences between birth and death timestamps. That means that the times $t_{i}$ are time offsets; time 0 is the birth time for all lines. For example, if we have a line with a lifespan from $t_{i}$ to $t_{j}$ we take for its death time the difference $t_{j}-t_{i}$. For the lines and the tokens that are right censored, we assign as their death timestamp the latest timestamp in the project. We also flag them as being alive, which means that in the Kaplan-Meier estimation their lifespan will be taken to be at least until the latest project timestamp. Note that we do not have censoring due to other causes, for example, a line being "lost" somewhere in the project's timeline, without being able to follow it up.

The function $S(t)$ is stepwise, with constant values between the different $t_{i}$. It estimates the survival function of a data set, which is formally defined as follows: $S(t)$ is the probability that an individual (line of code in our case) survives longer than a time $t$ :

$$
S(t)=P(T>t)
$$


In the above, $T$ is a variable indicating the time of death. We would also like to know what is the risk of dying at $t$. For this we have to turn to the hazard function, or hazard rate, $h(t)$, which is the rate at which an individual that has made it to time $t$ will die within an interval $\Delta t$ that tends to zero:

$$
h(t)=\lim _{\Delta t \rightarrow 0} \frac{P(t \leq T<\Delta t \mid T \geq t)}{\Delta t}
$$

We have three alternative hypotheses regarding the hazard function:

- Individuals run the same, constant, risk of death at each time $t$.

- Individuals run a higher risk of death when they are young; these are populations whose demographics are characterized by high infant mortality.

- Individuals run a higher risk of death when they are old; these are populations whose frailty increases with age (senescence).

To test these hypotheses we will check whether the hazard function for lines of code follows a Weibull distribution, a standard parametric model in survival analysis that has been used widely in science and engineering (Padgett, 2011). The Weibull distribution specifies the following hazard rate, with two parameters $\lambda>0, \alpha>0$ :

$$
h(t ; \lambda, \alpha)=\alpha \lambda t^{\alpha-1}
$$

The corresponding survival function is:

$$
S(t ; \lambda, \alpha)=e^{-\lambda t^{\alpha}}
$$

The parameter $\alpha$ is called the shape parameter and the parameter $\lambda$ is called the scale parameter. Together they determine the form of the corresponding Weibull probability density function $f(t ; \lambda, \alpha)=$ $\alpha \lambda t^{\alpha-1} e^{-\lambda t^{\alpha}}$. The parameter $\lambda$ stretches or contracts the distribution along the $x$ axis. There are three different cases for the parameter $\alpha$ :

- If $\alpha<1$, the hazard rate decreases over time.

- If $\alpha=1$, the hazard rate is constant.

- If $\alpha>1$, the hazard rate increases with time.

The three alternatives for $\alpha$ mirror the three hypotheses we want to check and can be the basis of the statistical analysis of the code aging process.

\section{RESULTS AND DISCUSSION}

RQ1 The Kaplan-Meier estimate provided the median lifespan for the projects we examined. This is the point in time at which 50\% of the population has died. Figure 3 shows the Kaplan-Meier survival functions for all projects, in increasing order.

The minimum line median lifespan, at 0.000105 years (about 0.9 hours), is for the project HandBrake; the maximum line median lifespan, at 10.03 years, is for collectd, while for torque and boto the lifespan could not be calculated, because not enough lines had died by the end of the data collection period to be able to get to the $50 \%$ point. We investigated the extremely low value for HandBrake. It appears that the project features large commits and incorporates the entire Mac Sparkle framework within the repo.

Turning to tokens, the minimum median lifespan was for odoo at 0.02 years. There were four projects for which no median lifespan could be calculated: thrift, $m p c$-hc, boto, collectd, torque. The maximum median lifespan that could be calculated was for docuwiki, at 12.33 years.

Taking all line results together, the median of the median lifespans is at 2.37 years, while the $25 \%$ percentile is at 1.54 years and the $75 \%$ percentile is at 4.25 years. For tokens, the corresponding median is 2.93 years, the $25 \%$ percentile 1.67 years, and the $75 \%$ percentile 5.36 years. These results indicate that lines and their individual tokens are durable rather than perishable, with lifespans measured in years rather than days. Figure 4 shows the histograms of the median lifespans. 


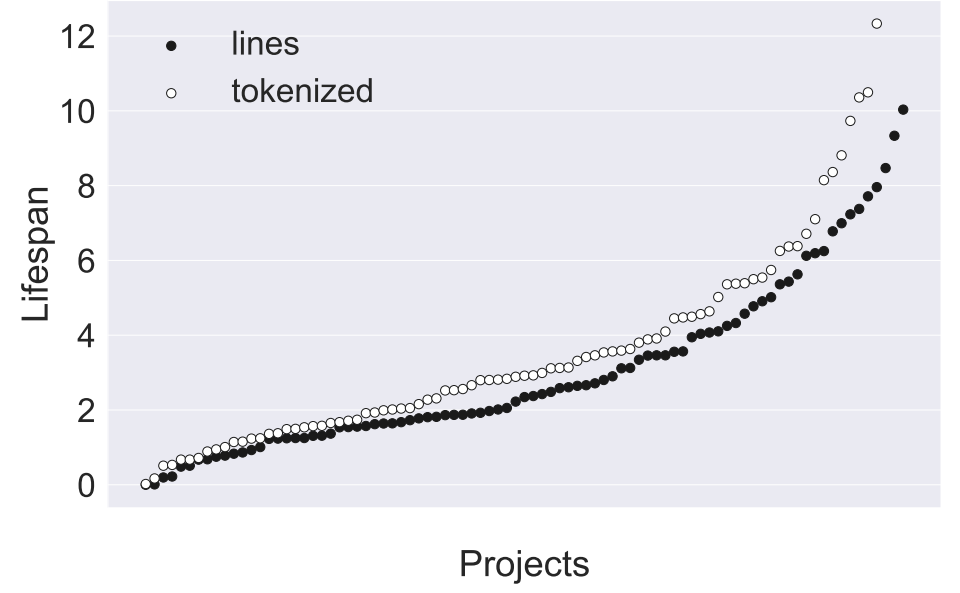

Figure 3. Kaplan-Meier median lifespan estimates

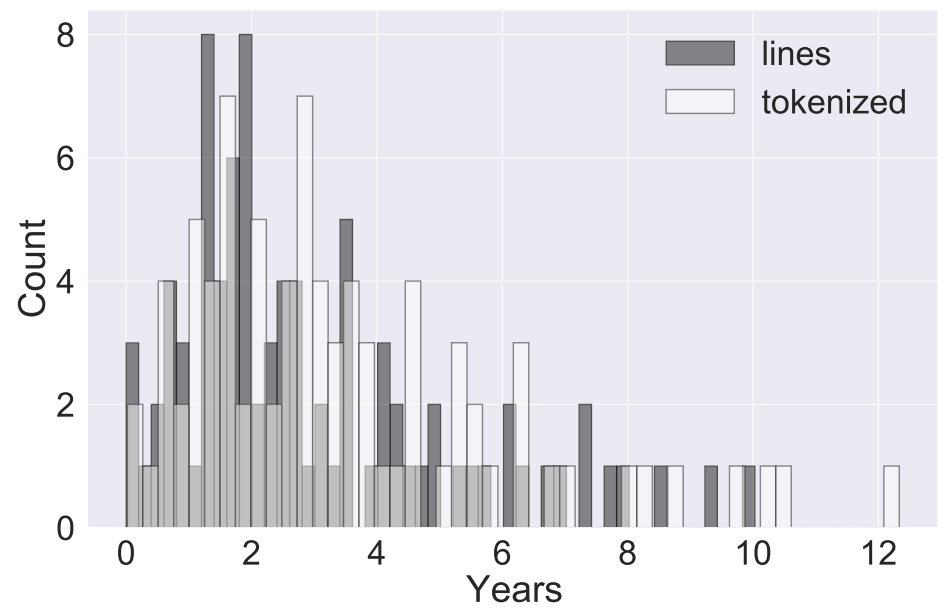

Figure 4. Histogram of median lifespans 


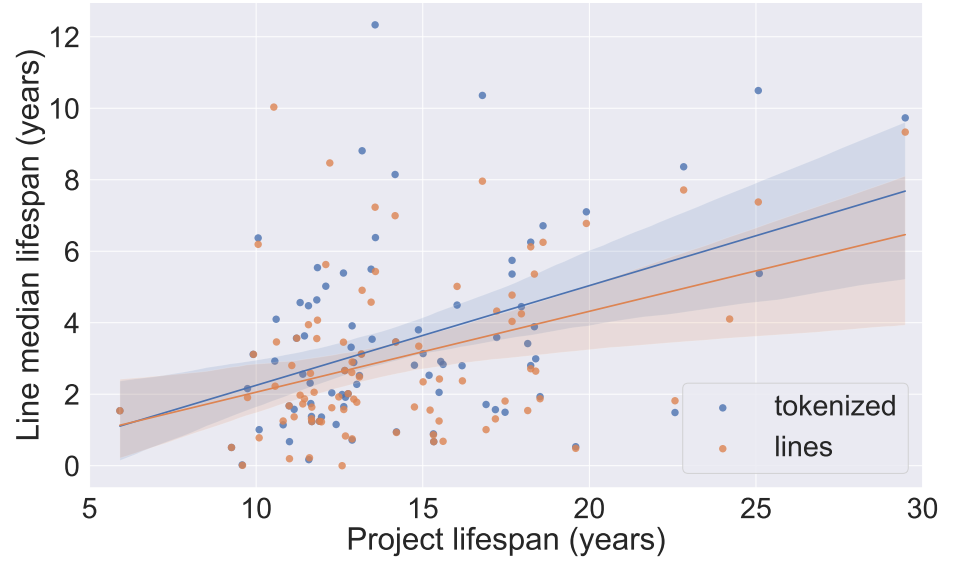

Figure 5. Line median lifespan on project lifespan

The growth of projects is punctuated with bursts of additions and deletes; these occur when a large body of code is imported or removed en masse from the project. We examined whether the estimates would change if we remove outliers. We therefore carried out the same statistics after removing the lines and tokens that where introduced in commits that were in the top $1 \%$ of commit size in every project. The line median lifespan moved to 2.54 years, an increase of $7.17 \%$; the token median lifespan moved to 3.18 years, an increase of $8.53 \%$. That is not trivial, but it does not change the overall picture.

To determine whether the differences in the medians between the line-oriented and the tokenized data can be explained away by chance, we carried out a Wilcoxon signed-rank test. The null hypothesis was that the two median populations come from the same distribution. The test allowed us to reject the null hypothesis with high probability ( $p$-value close to zero). It follows that code tokens lead longer lives than code lines; after all, every token that changes affects the line in which it belongs, but the opposite does not hold.

As project lifespans vary, the variability of code lifespans may be explained by the variability of project lifespans: code in longer-lived projects may live longer than code in younger projects. To investigate that, we performed correlation tests between median line lifespans and project lifespans. The Spearman correlation test for lines produced $\rho=0.29(p<0.01)$, which indicates a slight monotonically increasing relationship between median life lifespan and project lifespan. The Pearson correlation test produced $r=0.39(p \ll 0.01)$; the difference with the Spearman result can be explained if the relationship is monotonically increasing, but not linear. For tokenized data, the correlation was a bit stronger, with Spearman $\rho=0.37(p \ll 0.01)$ and Pearson $r=0.44(p \ll 0.01)$. Figure 5 shows a scatterplot of line median lifespans and project lifespans with a regression line; the regression coefficient for lines is 0.23 and for tokens is 0.28 . In all, although the effect of project age is statistically significant, its effect on the longevity of code is small.

RQ2 Moving beyond the estimates of median line lifespan, we checked the three hypotheses on hazard rates by fitting a Weibull distribution to each project's data. The fit was performed on the full line data of each project; we are interested in the fitted Weibull $\alpha$ parameter that controls the shape of the distribution and therefore the evolution of the hazard rate. The results of the fit showed that for all projects the $\alpha$ parameter is less than one, indicating a process with high infant mortality. Figure 6 shows the Weibull fitted distributions for all projects, each line being a project. The median of $\alpha$ is 0.52 , while the $25 \%$ percentile is 0.43 and the $75 \%$ percentile 0.63 . The situation is almost the same if we do the same analysis for tokens. Two projects have $\alpha \geq 1$ (by a whisker, ojs with $\alpha=1.01$ and $x m b c$ with $\alpha=1.07$ ). The median is 0.53 , the $25 \%$ percentile 0.43 and the $75 \%$ percentile 0.70 .

From the above it follows that young lines run higher risks. Old lines die as well, but younger ones at higher rates. This suggests a software development process where lines that are introduced into the code base of the project are subject to more change pressures. A line that has just been committed may not have been as thoroughly tested as older lines; it may need to be modified to accommodate factors that had not been foreseen; lines just added may impact more those recently introduced than parts of the older code base. 


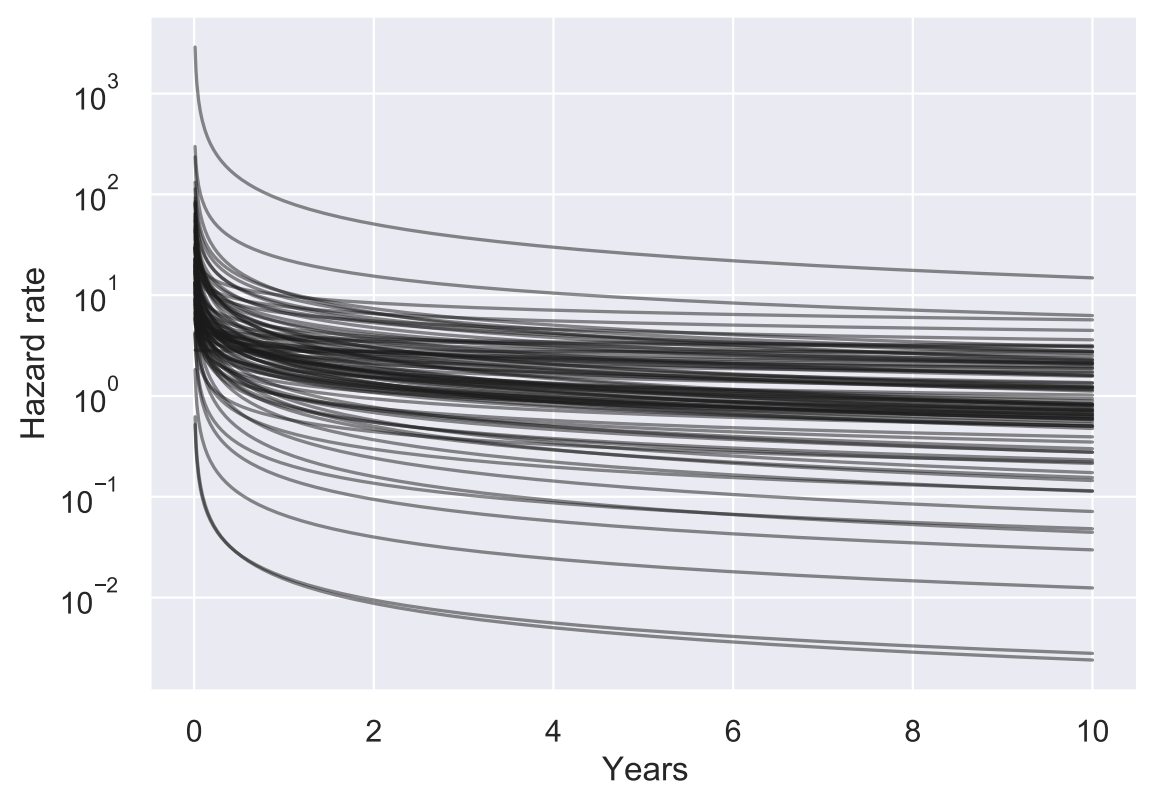

Figure 6. Hazard rates of lines of code per project.

Conversely, old lines seem to have proved their mettle. They have survived a long time and they are less likely to suffer changes than young lines. In a more negative light, old lines may gain a "don't touch" status, where developers are wary to change anything that works, which therefore lives on.

Whichever may hold, that a line lives on because it is really valuable or because nobody dares to change it, developers should be aware that they work for the long term. A line of code may live for years, well beyond the developers' involvement with a project or their ability to remember the rationale behind a cryptic choice. Consequently, our findings provide one more reason for writing clear and well-documented code.

Our findings also support the need to manage and perform what have been called anti-regressive changes (Lehman, 1978) to the software (effort required to combat the growth in complexity) in order to avoid the accumulation of technical debt (Kruchten et al., 2012). Code lines that live long are likely to become out of sync with respect to the software's evolving architecture, design, APIs, third-party libraries, language standards, as well as coding conventions and practices. As we have shown, such lines are not very likely to go away. Consequently, it is required to find those code lines that need care and bring them up to scratch. This is typically accomplished through the detection of code smells and the corresponding refactoring of code (Fowler, 2000).

RQ3a We investigated whether particular features of lines are conducive to more changes. We ran a linear multiple regression model for each project with the lifespan of a line as the dependent variable and as independent variables the length of the line, the indentation, the number of strings in the line, whether it (or part of it) is a comment, the number of commas, the number of brackets, the number of access operators (method and pointer), the number of assignments, the number of scope delimiters, the number of array accesses, and the number of logical operators. The elements we tested point to code smells or other code features that may make the code less stable, affecting its lifetime. We selected the aforementioned features for the following reasons. A large number of brackets may indicate complicated conditions or expressions, or long statements, which are a known code smell (Sharma et al., 2016). A large number of commas may indicate a long parameter list smell (Mäntylä and Lassenius, 2006). Strings may indicate the entanglement of presentation elements with business logic (Nguyen et al., 2012).

The results showed a very low fit $\left(R^{2}<0.1\right)$ for all projects, apart from canu with $R^{2}=0.44$, HandBrake, with $R^{2}=0.29$, and pyparallel, with $R^{2}=0.11$. The regression coefficients were found with very small $p$ values, which indicates that the influence they have on the lifespan cannot be explained away by chance, but the whole linear model, and therefore each particular predictor in it, accounts for a tiny part of lifetime variance. 
RQ3b To conduct a similar analysis for tokens, we divided tokens in four types: identifiers (391 million), numbers (91 million), keywords (112 million), and other tokens (mainly operators and punctuation-723 million). We ran pairwise Mann-Whitney $U$ tests between the lifetimes of different token types for each project. The distributions of the lifetimes of token types per project are different in the vast majority of projects (ranging from the distributions of $86 / 89$ projects for identifiers versus other tokens to the distributions of $82 / 89$ projects for keywords versus numbers). However, when we take the medians of the lifetimes of different token types for all projects, their distributions are then all indistinguishable. As for lines, we could not determine that some particular types of tokens are associated with longer lifetimes across projects.

RQ3C A different factor that may influence the lifespan of a line is the committer who enters, alters, or deletes a line. We examined possible correlations between the lifespan and the number of developer commits in the project and between the experience and the tenure of the developer in the project. For this we looked at commits in the middle year of the examined period (2012), thus providing at least 4.5 years time to gather line and developer data and then another 4.5 for lines to disappear. We used the number of a developer's project commits until each examined commit as a measure of a developer's experience, and the difference in time between the developer's first project commit and the examined one as a measure of the developer's tenure in the project. We carried out both Spearman and Pearson correlation tests to examine the relationship between line lifetime and the experience and tenure of the developer who added the line. We could not identify a single rule across projects. In some projects, committer activity and tenure appear to be positive correlated with line lifespan, in other projects they appear to be negative correlated, and in most projects the correlation seems to be weak: the median is close to zero. The situation changes when we examine the lifetime of lines versus the experience and tenure of the author who removed them: we find that the lifetime is positively correlated with developer experience, that is, more experienced developers remove longer-lived lines. The median of the correlation of line lifetime and developer experience across projects is 0.27 (Spearman) and 0.24 (Pearson) for $p<0.05$; for the correlation of line lifetime and developer tenure the medians are 0.42 (Spearman) and 0.33 (Pearson) for $p<0.05$.

Alternatively, the above can mean that it takes experienced developers to remove a long-lived line, bringing us back to the "don't touch" status. The "don't touch" status also hints at a different facet of the way lines are handled. Could it be that lines are more likely to be changed or deleted by the same developer who entered them into a project in the first place, rather than by a different committer? We contrasted, for each project, the lifetimes of lines that are changed by the same developer against those that are changed by a different one. The two distributions are different (checking with the Mann-Whitney $U$ test) for all projects except drush and grails-core. In most projects, the median lifetime of lines removed by the same author who entered them is less than the median lifetime of lines removed by a different author $(81 / 89)$; and similarly for the means $(85 / 89)$. In short, lines are more likely to be touched by their original author (see also Figure 7).

RQ3d We investigated whether project size affects the longevity of lines and tokens. We checked the number of lines and the number of tokens for all commits, using both Pearson and Spearman correlations. We found only slight positive correlations, $r=0.35(p<0.01)$,for the Pearson correlation (but not the Spearman) for both the number of lines and the number of tokens in a project. Of course, the size of the project may be related to its age and indeed the results are concordant with our preceding investigation on code lifespans and project lifespans.

RQ3e Turning to programming languages, although we expected to find greater lifespans in languages with features that promote modularity, we did not detect that. Table 2 shows that median lifetime estimates over projects grouped by programming language (excluding a single project in $\mathrm{C \#}$ ). If anything, we see that, for instance, $\mathrm{C}$ exhibits greater lifespans than $\mathrm{C}++$. However, note that none of the differences between programming languages was statistically significant at the 0.01 level using the Mann-Whitney $U$ test. 


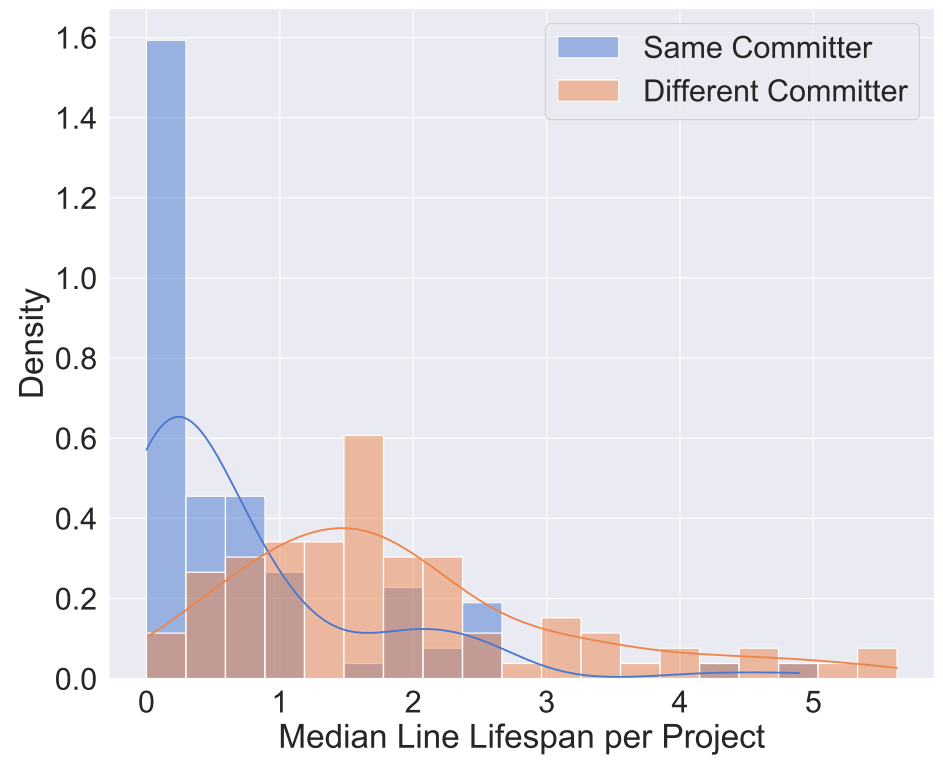

Figure 7. Per project lifetime medians for same and different committers

Table 2. Languages and Kaplan-Meier (KM) Estimates

\begin{tabular}{lccc}
\hline Language & \# Projects & $\begin{array}{c}\text { Median } \\
\text { Line KM }\end{array}$ & $\begin{array}{c}\text { Median } \\
\text { Token KM }\end{array}$ \\
\hline PHP & 16 & 1.93 & 2.42 \\
C++ & 17 & 1.86 & 2.81 \\
Python & 12 & 2.75 & 3.61 \\
Java & 18 & 2.45 & 2.74 \\
C & 25 & 2.80 & 3.63 \\
\hline
\end{tabular}

\section{THREATS TO VALIDITY}

\section{Internal Validity}

Thankfully, by basing our study on historical data, many threats that typically appear in evolving experiments, such as design contamination, experimental mortality, and maturation, can be ruled out. The main remaining threats are associated with confounding factors, noise in the data, commit granularity, file differencing, and statistical methods.

An important consideration is that the independent variable we used in our study, a code line's age, can encompass many other variables. Specifically with the passage of time, the number of faults in a line will decrease as these are winnowed out (Ozment and Schechter, 2006), the developers' familiarity will increase as they read it again and again, and the line's afferent couplings may increase as other code may depend on its elements.

Another factor is noise in the data we used. Although we were careful to include (through simple measures) in our study what has been termed engineered software projects (Munaiah et al., 2017), we cannot exclude the possibility that the underlying commit data contain infelicities that may influence our results. These include the addition or removal of large third-party software systems, wrongly set commit dates, history rewrites performed after the code was written, and errors introduced when one type of repository (e.g. CVS) gets converted into Git.

Third-party code changes can have a significant effect on software evolution. Gall et al. (1997) conducted an empirical study on a large telecommunication switching system, and identified important differences in the patterns of software evolution over time of the whole system versus its subsystems. A similar strategy of separately examining the growth of subsystems of large software projects has been followed by González-Barahona et al. (2014) and by González-Barahona et al. (2009). Other studies of software evolution have also identified the ripples caused by the inclusion or removal of third-party 
components (Robles et al., 2005), and some, such as the one by Hatton et al. (2017), have attempted to address the issue by filtering them out. As we have not performed such filtering, these changes may affect our reported results. On the other hand, filtering introduces another threat to validity due to the subjective nature of the required decisions or parameters.

A related factor is the granularity of the studied commits. Our study is missing many intermediary commits, first because we removed about $32 \%$ through history simplification, and second because many others may have occurred in third-party repositories and then pushed upstream as a single commit (German et al., 2016). One could argue that the effects of history simplification should cancel out: lines would on average appear later and also disappear later. Nevertheless, to quantify the effect of history simplification, we measured the interval between commits in both the complete tree and the simplified longest path. As expected, the longer time paths upstream from merges in the complete tree, which were simplified away in the linear path, gave the tree a longer interval between commits (a median of 40 minutes) than its longest path (27 minutes). However, the difference between the median value of the two intervals (13 minutes) is five orders of magnitude smaller than the line lifespan we report, making any effect negligible.

The use of Git to list the differences between two file versions is also a threat. First, the employed file difference algorithm (Myers, 1986) will display a movement of code as a deletion and an insertion. Then, relatively minor changes, such as the renaming of an identifier, will appear as line deletions and insertions, which may skew the results toward higher infant mortality. We examined the effect of these two issues through methods described in the sections on the analysis of individual tokens and moved lines. Also, the detection of file renaming and copying is based on a heuristic and a threshold. We used the default thresholds, only increasing the number of files that would be checked for copies; there may conceivably be better values to use.

A related issue is that our investigation focuses on individual code lines. We do not take context into account. A line that is moved from one place to the next impacts both places and may cause cascading changes in the lifespans of other lines. However, the same applies in traditional survival analysis: deaths may be related to other deaths (e.g., via disease); we are interested in the lifespan of lines, no matter the relationships that may exist between them.

Given that we used custom-developed tools to track the birth and death of lines of code, human error is an inevitable factor. We tried to minimize this through numerous test cases, manual verification, and the use of internal consistency checks.

Turning to our statistical methods, we have used two statistical techniques to answer two different, but related questions. We used the Kaplan-Meier estimator to investigate the median lifespan of code in projects, and a Weibull process to investigate the overall aging process. The Kaplan-Meier estimator provided us with approximations of the survival functions, while the Weibull fit gave us approximations of the hazard functions. We assumed that the hazard rate is characterized by a Weibull function, because the Weibull distribution is a popular model for several related process such as component failure rates and Weibull covers different aging processes depending on the value of $\alpha$. Moreover, we are not interested in the exact values of the parameters of the Weibull distribution, but in the relation of $\alpha$ to 1 , where we found consistent results. The remarkable agreement in the shape of the Weibull distributions among many diverse projects (Figure 6) leads us to believe that our findings are reproducible and generalizable. We examined whether the lognormal distribution, which is also often used in failure models, would be a better fit for our data. To compare the two models, Weibull and lognormal, we used the Akaike Information Criterion (AIC), defined as AIC $=2 k-2 \log (\hat{L})$, where $k$ is the number of parameters of the model and $\hat{L}$ is the maximum likelihood of the model. A lower AIC value corresponds to a better fit, as this maximizes the goodness of fit, given by the log-likelihood, but penalizes the complexity of the model, given by the number of parameters. We found overwhelmingly that the Weibull distribution was a better fit. Only five projects had better fit with the lognormal distribution when we examined the lines, and six projects had better fit with the lognormal distribution when we examined the tokens (four projects were the same). We used the Python lifelines package for calculating the estimates and comparing the distributions (Davidson-Pilon et al., 2020).

\section{External Validity}

The generalizability of our findings is threatened by our choice of analyzed projects. Although we included projects from diverse development communities, written in numerous programming languages, and serving many different application areas, we cannot claim that our choice represents adequately all 
software development. In particular, we feel that our sample excludes or underrepresents the following software types: small software projects, projects developed with tightly managed or formal processes, proprietary and bespoke systems, projects written in programming languages not favoured by the open source community, and systems that target specific application domains rather than the provision of systems infrastructure.

More importantly, our findings are based on large, successful projects that have run for several years. There are many more projects that are discontinued after a short period of time, for any reason. All lines of code in these projects freeze at an early stage of what could have been a longer period of evolution. Therefore our findings cannot be generalized to all software development- this would be an instance of survival bias, reaching conclusions for all the population based only on the characteristics of the survivors. That said, people usually aspire to create successful, long-lasting projects, so our findings are pertinent for those projects that want to achieve longevity.

\section{RELATED WORK}

All living beings degenerate and die with age. The origin of senescence, however, remains an unsolved problem for biologists (Kirkwood and Austad, 2000). Likewise, many software components evolve, age, and are eventually removed or replaced. This section presents related work regarding the fields of software evolution, aging, and decay, and records empirical studies that use the statistical method of survival analysis (Elandt-Johnson and Johnson, 1990; Klein and Moeschberger, 2003).

The process of software evolution refers to the modification and adaptation of software programs so that programs can survive as their environment changes. The software evolution laws of Lehman (1980) describe the constraints practitioners should take into account to continuously adapt actively used software systems. A detailed literature review regarding Lehman's software evolution laws has been conducted by Herraiz et al. (2013). Many empirical studies focus on predictive models of software projects' evolution at macroscopic scale. Relevant studies have looked at long-term sustainability factors in the evolution of LibreOffice (Gamalielsson and Lundell, 2014), the change of program dependencies in the Apache ecosystem (Bavota et al., 2013), and the early identification of source code evolution pattern in open source projects (Karus, 2013). Additionally, many empirical studies examine software evolution at microscopic level, considering the evolution of source-code elements such as methods. In particular, Bevan et al. (2005) developed Kenyon, which supports different types of stratigraphic software evolution research, ranging from code feature evolution to dependency graph-based maintenance. Zimmermann (2006) presented APFEL for fine-grained processing of source code elements such as tokens, method calls, exceptions, and variable usages. Hata et al. (2011) introduced Historage, which provides entire histories of fine-grained entities in Java, such as methods, constructors, and fields. This tool has been applied to quantitatively evaluate the remaining change identification of open source software projects.

The term software aging was coined by Parnas (1994) and refers to the idea that programs, like people, are getting old. According to Parnas, software aging happens for two reasons: 1) software fails to adapt to changing needs, and 2) software changes but in an inappropriate way (addition of bad fixes and features). Given, however, that it is infeasible for developers to prevent software evolution and, consequently, software degradation, researchers attempt to limit program damages by predicting the software's lifetime and inventing rejuvenation approaches (Karus and Dumas, 2012; Li et al., 2011; Qin et al., 2005; Robillard and Murphy, 2007; Salfner et al., 2010). In the field of software aging, empirical studies have been conducted on the identification of aging trends. In particular, Robles et al. (2005) found that a system becomes "old" when it turns five. The authors also defined the absolute 5-year aging index to compare the relative aging of different projects. Finally, Cotroneo et al. (2013) developed an approach that predicts the location of aging-related bugs using software complexity metrics and machine learning algorithms. They found that the most significant signs of software aging manifest themselves as: leaked memory, unterminated threads, unreleased files and locks, numerical errors, and disk fragmentation.

As software evolves, developers should overcome software erosion by fighting software decay. A significant research body is also devoted to this field. Eick et al. (2001) used the term code decay to describe the situation where evolving software increasingly hinders software maintenance. The authors, also, proposed measurements (code decay indices) as decay predictors. For their study, they statically analyzed millions of lines of a fifteen-year old telephone switching software system. Similarly to our work, the authors tracked added and deleted source code lines. However, they did not use survival analysis and they examined a single project to find particular code decay factors. Additionally, Arisholm and 
Sjøberg (2000) proposed a framework for the empirical assessment of changeability decay and Araújo et al. (2012) built a software decay model regarding software deterioration causes.

Extensive work has been done on identifying and tracking code changes. Kim and Notkin (2006) were the first that defined the problem of matching code elements between two program versions based on syntactic and textual similarity. To compute the difference between two programs several tools have been implemented. Canfora et al. (2007) developed a technique that combines Space Vector Models and the Levenshtein edit distance for finding CVS/SVN differences that occur due to line additions or deletions, as well as due to line modifications. Furthermore, the LHDiff tool implements languageindependent techniques to track how source code lines evolve across different versions of a software system (Asaduzzaman et al., 2013b). The tool uses the simhash technique together with heuristics. In addition, the GumTree tool identifies edits in scripts when moving code in version repositories (Falleri et al., 2014). This tool is based on a abstract syntax tree (AST) differencing algorithm. The ChangeDistiller is another differencing tool that is based on a tree differencing algorithm for fine-grained source code change extraction (Fluri et al., 2007). To represent how lines evolve over time in source code version repositories researchers have also used annotation graphs (Zimmermann et al., 2006). More recently, Servant and Jones (2017) proposed a fine-grained model based on optimizing the Levenshtein difference between lines of successive versions. Finally, CVSscan is a visual tool for representing software evolution based on the tracking of line-based source code changes extracted by using Unix's diff (Voinea et al., 2005). The lifetime tool we present here, balances computational cost with accuracy by processing a series of git diff outputs and uses a state machine for the parsing of their output.

Other researchers have also employed the tools of survival analysis in software (Elandt-Johnson and Johnson, 1990; Klein and Moeschberger, 2003). Sentas et al. (2008) developed a statistical framework based on survival analysis and the Kaplan-Meier estimator (E. L. Kaplan, 1958) to predict the duration of software projects and the factors that affect it. The authors applied their approach on proprietary software projects taking into account industrial factors that have an impact on a project's lifetime. They found that the median duration of the examined projects is fourteen months. Similarly, Samoladas et al. (2010) applied survival analysis on 1,147 open source projects to forecast software evolution trends. The authors observed that projects that existed more than ten years ago continue to evolve. Comparably, our insights confirm that code in long-lived projects lives longer. Scanniello (2011) used the Kaplan-Meier estimator on five software projects (at method level) to investigate how dead code affects software evolution.

Our finding regarding the lower hazard of older lines is mirrored by Zheng et al. (2008) who report that in Gentoo Linux packages, a network graph new node is connected to an old node with a probability that depends not only on the degree but also on the age of the old node. Other survival analysis studies include the one by Claes et al. (2015) on the longevity of Debian packages with conflicts and the one by Goeminne and Mens (2015) on the survival and influence of five Java database frameworks. To the best of our knowledge, this paper is the first work that uses survival analysis to track the birth and death of code lines and tokens over periods that span decades, and presents a theoretical and statistical model regarding the aging process of code lines.

Another research strand where the study of the evolution of fine-grained code elements is related to includes genetic improvement. Genetic improvement (GI) uses automated search (i.e., optimization and machine learning techniques) in order to improve existing software. Typically GI involves making small changes or edits (also known as mutations) in source-code elements (i.e., lines of code or tokens) to improve existing software. Topics covered by GI research include program transformation, approximate computing, and program repair (Petke et al., 2018). As an example, Petke et al. (2014) apply GI with automated code transplantation, by mutating the code at the level of lines of source code, to improve software performance. Additionally, Barr et al. (2014) introduce the plastic surgery hypothesis, which states that changes to a codebase refer to source code elements that already exist in the codebase at the time of a change. Related work (Goues et al., 2013; Nguyen et al., 2013) considers repetitiveness of code changes (abstracted to abstract syntax trees) that is associated with the plastic surgery hypothesis. Furthermore, Martinez et al. (2014) consider changes that could be constructed based on existing snippets. Therefore, the study of the evolution of code elements, such as code lines or tokens that we take into account here, could help, in the future, in the guidance of software improvement based on evolutionary approaches. 


\section{CONCLUSIONS AND IMPLICATIONS}

When we began working on this study, we did not know whether code lines were durable or perishable and whether their demise was a result of infant mortality or senescence. By devising a method and implementing tools to follow source code lines from 89 revision control repositories from their birth to their demise we were able to arrive at the answers through the statistical analysis of 3.3 billion source code lifetime events. We found that code lines are durable with a median lifespan of about 2.3 years, the corresponding median for the tokens is 2.9 years, and that the associated hazard rate decreases over time following a Weibull distribution; i.e., young lines are more likely to be modified or deleted. We investigated whether line and token longevity are associated with particular line and token features, developer experience and tenure in a project, and programming language. Our results did not show strong patterns, indicating that line and token longevity may be the result of a complex interaction of various, potentially context specific, factors. Project age and project size had a small correlation with code longevity.

On the practical front, our model, suitably calibrated, can provide input for estimating maintenance effort, while the corresponding tool could aid the management of technical debt and the risk assessment of software vulnerabilities. Our model derives statistical estimates of lifespan estimates and hazard rates, based on the source code of projects. They can be run on other projects, apart from the ones we used, to give the calibrated figures for them. Knowing how lines and token age (or churn) in a project may help in managing technical debt and risk assessment (Ozment and Schechter, 2006; Shin et al., 2010). For example, a large number of long-lived lines / tokens can be a sign of stability or "don't touch" status. Moreover, our regression model of lines lifespan Vs. project lifespan can be used against a particular project to gauge where it stands, or whether (perhaps problematically) it is an outlier. All these potential uses need to be empirically validated in future studies.

On the research front, the study of code evolution at the level of individual lines can be extended both theoretically and in empirical breadth and depth. On the theoretical side, significant work is required to establish the precise mechanisms underlying the observed hazard rate. Features we did not examine here, such as as the interplay of requirements, architecture, and syntax, might be worthy candidates for further study. Corresponding theories should then be empirically evaluated using our methods and tools. On the breadth side examining more, and more diverse, repositories will strengthen the generalizability of our findings.

Tying together this area's research and practical implications is the enticing quest to identify and control factors that $d o$ play a role in the lifetime of code elements. Once these are nailed down, software engineering practices can be correspondingly adjusted so as to reduce potentially wasteful effort by delivering code lines with longer lifespans. This line of research can lead to a new promising and exciting avenue for improving the efficiency of software development.

\section{Acknowledgements}

The authors thank Alexander Chatzigeorgiou for his valuable and timely feedback. This work's first author thanks Michiel van Genuchten and Les Hatton for their fruitful collaboration on software growth modeling.

\section{REFERENCES}

Albrecht AJ, Gaffney JE (1983) Software function, source lines of code, and development effort prediction: A software science validation. IEEE Transactions on Software Engineering SE-9(6):639-648, doi: 10.1109/TSE.1983.235271

Allamanis M, Barr ET, Devanbu P, Sutton C (2018) A survey of machine learning for big code and naturalness. ACM Comput Surv 51(4):81:1-81:37, doi: 10.1145/3212695

Alon U, Zilberstein M, Levy O, Yahav E (2019) Code2Vec: Learning distributed representations of code. Proc ACM Program Lang 3(POPL):40:1-40:29, doi: 10.1145/3290353

Araújo MAP, Monteiro VF, Travassos GH (2012) Towards a model to support in silico studies of software evolution. In: Proceedings of the 2012 ACM-IEEE International Symposium on Empirical Software Engineering and Measurement, ESEM '12, pp 281-289, doi: 10.1145/2372251.2372303 
Arisholm E, Sjøberg DIK (2000) Towards a framework for empirical assessment of changeability decay. Journal of Systems and Software 53(1):3-14, doi: 10.1016/S0164-1212(00)00003-0

Asaduzzaman M, Roy CK, Schneider KA, Di Penta M (2013a) LHDiff: A language-independent hybrid approach for tracking source code lines. In: ICSM 2013: 29th IEEE International Conference on Software Maintenance, IEEE, pp 230-239

Asaduzzaman M, Roy CK, Schneider KA, Penta MD (2013b) LHDiff: Tracking source code lines to support software maintenance activities. In: 2013 IEEE International Conference on Software Maintenance, pp 484-487, doi: 10.1109/ICSM.2013.78

Atkins DL, Ball T, Graves TL, Mockus A (2002) Using version control data to evaluate the impact of software tools: a case study of the Version Editor. IEEE Transactions on Software Engineering 28(7):625-637, doi: 10.1109/TSE.2002.1019478

Barnes JM, Pandey A, Garlan D (2013) Automated planning for software architecture evolution. In: 28th IEEE/ACM International Conference on Automated Software Engineering (ASE '13), pp 213-223, doi: 10.1109/ASE.2013.6693081

Barr ET, Brun Y, Devanbu P, Harman M, Sarro F (2014) The plastic surgery hypothesis. In: Proceedings of the 22nd ACM SIGSOFT International Symposium on Foundations of Software Engineering, Association for Computing Machinery, New York, NY, USA, FSE 2014, pp 306-317, doi: 10.1145/ 2635868.2635898

Bavota G, Canfora G, Penta MD, Oliveto R, Panichella S (2013) The evolution of project interdependencies in a software ecosystem: The case of Apache. In: Proceedings of the 29th IEEE International Conference on Software Maintenance, IEEE Computer Society, Washington, DC, USA, ICSM '13, pp 280-289, doi: 10.1109/ICSM.2013.39

Bevan J, Whitehead EJ, Kim S, Godfrey M (2005) Facilitating software evolution research with Kenyon. In: Proceedings of the 10th European Software Engineering Conference Held Jointly with 13th ACM SIGSOFT International Symposium on Foundations of Software Engineering, Association for Computing Machinery, New York, NY, USA, ESEC/FSE-13, pp 177-186, doi: 10.1145/1081706. 1081736

Breivold HP, Crnkovic I, Larsson M (2012) A systematic review of software architecture evolution research. Information and Software Technology 54(1):16-40, doi: 10.1016/j.infsof.2011.06.002

Buse RP, Weimer WR (2008) A metric for software readability. In: Proceedings of the 2008 International Symposium on Software Testing and Analysis, ACM, New York, NY, USA, ISSTA '08, pp 121-130, doi: $10.1145 / 1390630.1390647$

Canfora G, Cerulo L, Penta MD (2007) Identifying changed source code lines from version repositories. In: Proceedings of the 4th International Workshop on Mining Software Repositories, IEEE Computer Society, Washington, DC, USA, MSR '07, p 14, doi: 10.1109/MSR.2007.14

Claes M, Mens T, Di Cosmo R, Vouillon J (2015) A historical analysis of Debian package incompatibilities. In: Proceedings of the 12th Working Conference on Mining Software Repositories, IEEE Press, Piscataway, NJ, USA, MSR '15, pp 212-223

Cotroneo D, Natella R, Pietrantuono R (2013) Predicting aging-related bugs using software complexity metrics. Performance Evaluation 70(3):163-178, doi: 10.1016/j.peva.2012.09.004, special Issue on Software Aging and Rejuvenation

Davidson-Pilon C, Kalderstam J, Jacobson N, sean reed, Kuhn B, Zivich P, Williamson M, AbdealiJK, Datta D, Fiore-Gartland A, Parij A, WIlson D, Gabriel, Moneda L, Moncada-Torres A, Stark K, Gadgil H, Jona, Singaravelan K, Besson L, Peña MS, Anton S, Klintberg A, GrowthJeff, Noorbakhsh J, Begun M, Kumar R, Hussey S, Golland D, jlim13 (2020) Camdavidsonpilon/lifelines: v0.25.6. doi: 10.5281/zenodo.4136578, URL https: / / doi .org/10.5281/zenodo.4136578 
E L Kaplan PM (1958) Nonparametric estimation from incomplete observations. Journal of the American Statistical Association 53(282):457-481, URL http: / / www. jstor.org/stable/2281868

Eick SG, Graves TL, Karr AF, Marron JS, Mockus A (2001) Does code decay? assessing the evidence from change management data. IEEE Transactions on Software Engineering 27(1):1-12, doi: 10.1109/ 32.895984

Elandt-Johnson RC, Johnson NL (1990) Survival models and data analysis. Wiley

Falleri JR, Morandat F, Blanc X, Martinez M, Monperrus M (2014) Fine-grained and accurate source code differencing. In: Proceedings of the 29th ACM/IEEE International Conference on Automated Software Engineering, ACM, New York, NY, USA, ASE '14, pp 313-324, doi: 10.1145/2642937.2642982

Fluri B, Wuersch M, PInzger M, Gall H (2007) Change distilling: Tree differencing for fine-grained source code change extraction. IEEE Transactions on Software Engineering 33(11):725-743, doi: 10.1109/TSE.2007.70731

Fowler M (2000) Refactoring: Improving the Design of Existing Code. Addison-Wesley, Boston, MA

Gall H, Jazayeri M, Klosch RR, Trausmuth G (1997) Software evolution observations based on product release history. In: 1997 Proceedings International Conference on Software Maintenance, pp 160-166, doi: 10.1109/ICSM.1997.624242

Gamalielsson J, Lundell B (2014) Sustainability of open source software communities beyond a fork: How and why has the LibreOffice project evolved? Journal of Systems and Software 89:128-145, doi: 10.1016/j.jss.2013.11.1077

van Genuchten M, Hatton L (2013) Metrics with impact. IEEE Software 30(4):99-101, doi: 10.1109/MS 2013.81

German DM, Adams B, Hassan AE (2016) Continuously mining distributed version control systems: an empirical study of how Linux uses Git. Empirical Software Engineering 21(1):260-299, doi: $10.1007 / \mathrm{s} 10664-014-9356-2$

German DM, Adams B, Stewart K (2019) cregit: Token-level blame information in git version control repositories. Empirical Software Engineering doi: 10.1007/s10664-019-09704-x

Giger E, Pinzger M, Gall HC (2011) Comparing fine-grained source code changes and code churn for bug prediction. In: Proceedings of the 8th Working Conference on Mining Software Repositories, ACM, New York, NY, USA, MSR '11, pp 83-92, doi: 10.1145/1985441.1985456

Godfrey MW, Tu Q (2000) Evolution in open source software: A case study. In: Proceedings of the 2000 International Conference on Software Maintenance, IEEE, ICSM '00, pp 131-142

Goeminne M, Mens T (2015) Towards a survival analysis of database framework usage in Java projects. In: Proceedings of the 31st IEEE International Conference on Software Maintenance and Evolution, ICSME '15, pp 551-555, doi: 10.1109/ICSM.2015.7332512

González-Barahona JM, Robles G, Michlmayr M, Amor JJ, German DM (2009) Macro-level software evolution: a case study of a large software compilation. Empirical Software Engineering 14(3):262-285, doi: $10.1007 / \mathrm{s} 10664-008-9100-\mathrm{x}$

González-Barahona JM, Robles G, Herraiz I, Ortega F (2014) Studying the laws of software evolution in a long-lived FLOSS project. Journal of Software: Evolution and Process 26(7):589-612, doi: $10.1002 / \mathrm{smr} .1615$

Gordon AD, Henzinger TA, Nori AV, Rajamani SK (2014) Probabilistic programming. In: Proceedings of the on Future of Software Engineering, ACM, New York, NY, USA, FOSE 2014, pp 167-181, doi: $10.1145 / 2593882.2593900$

Goues C, Forrest S, Weimer W (2013) Current challenges in automatic software repair. Software Quality Journal 21(3):421-443, doi: 10.1007/s11219-013-9208-0 
Gousios G, Spinellis D (2012) GHTorrent: Github's data from a firehose. In: Lanza M, Penta MD, Xie T (eds) 9th IEEE Working Conference on Mining Software Repositories (MSR), IEEE, pp 12-21, doi: 10.1109/MSR.2012.6224294

Gousios G, Spinellis D (2017) Mining software engineering data from GitHub. In: Proceedings of the 39th International Conference on Software Engineering Companion, IEEE Press, Piscataway, NJ, USA, ICSE-C '17, pp 501-502, doi: 10.1109/ICSE-C.2017.164, technical Briefing

Gousios G, Kalliamvakou E, Spinellis D (2008) Measuring developer contribution from software repository data. In: Proceedings of the 2008 International Working Conference on Mining Software Repositories, ACM, New York, NY, USA, MSR '08, pp 129-132, doi: 10.1145/1370750.1370781

Hata H, Mizuno O, Kikuno T (2011) Historage: Fine-grained version control system for Java. In: Proceedings of the 12th International Workshop on Principles of Software Evolution and the 7th Annual ERCIM Workshop on Software Evolution, Association for Computing Machinery, New York, NY, USA, IWPSE-EVOL '11, pp 96--100, doi: 10.1145/2024445.2024463

Hatton L, Spinellis D, van Genuchten M (2017) The long-term growth rate of evolving software: Empirical results and implications. Journal of Software: Evolution and Process doi: doi:10.1002/smr.1847

Heckel P (1978) A technique for isolating differences between files. Commun ACM 21(4):264-268, doi: $10.1145 / 359460.359467$

Herraiz I, González-Barahona JM, Robles G (2007) Towards a theoretical model for software growth. In: Proceedings of the 4th International Workshop on Mining Software Repositories, IEEE Computer Society, Washington, DC, USA, MSR '07, p 21, doi: 10.1109/MSR.2007.31

Herraiz I, Rodriguez D, Robles G, González-Barahona JM (2013) The evolution of the laws of software evolution: A discussion based on a systematic literature review. ACM Computing Surveys 46(2):28:128:28, doi: $10.1145 / 2543581.2543595$

Humphrey WS (1989) Managing the Software Process. Addison-Wesley, Reading, MA

Ince D, Hatton L, Graham-Cumming J (2012) The case for open program code. Nature 482:485-488, doi: 10.1038/nature10836

Jiang S, Armaly A, McMillan C (2017) Automatically generating commit messages from Diffs using neural machine translation. In: Proceedings of the 32Nd IEEE/ACM International Conference on Automated Software Engineering, IEEE Press, Piscataway, NJ, USA, ASE 2017, pp 135-146

Kan SH (2002) Metrics and Models in Software Quality Engineering, 2nd edn. Addison-Wesley Longman Publishing Co., Inc., Boston, MA, USA

Karus S (2013) Automatic means of identifying evolutionary events in software development. In: Proceedings of the 29th IEEE International Conference on Software Maintenance, IEEE, ICSM '13, pp $412-415$

Karus S, Dumas M (2012) Code churn estimation using organisational and code metrics: An experimental comparison. Information and Software Technology 54(2):203-211, doi: 10.1016/j.infsof.2011.09.004

Kechagia M, Devroey X, Panichella A, Gousios G, van Deursen A (2019) Effective and efficient API misuse detection via exception propagation and search-based testing. In: Proceedings of the 28th ACM SIGSOFT International Symposium on Software Testing and Analysis, ACM, New York, NY, USA, ISSTA 2019, pp 192-203, doi: 10.1145/3293882.3330552

Kim M, Notkin D (2006) Program element matching for multi-version program analyses. In: Proceedings of the 2006 International Workshop on Mining Software Repositories, ACM, New York, NY, USA, MSR '06, pp 58-64, doi: 10.1145/1137983.1137999

Kirkwood TB, Austad SN (2000) Why do we age? Nature 408(6809):233-238

Peerj Comput. Sci. reviewing PDF | (CS-2020:10:54298:1:1:CHECK 22 Dec 2020) 
Klein JP, Moeschberger ML (eds) (2003) Survival Analysis: Techniques for Censored and Truncated Data, 2nd edn. Springer

Kruchten P, Nord RL, Ozkaya I (2012) Technical debt: From metaphor to theory and practice. IEEE Software 29(6):18-21, doi: 10.1109/MS.2012.167

Lehman MM (1978) Programs, cities, students — limits to growth? In: Gries D (ed) Programming Methodology: A Collection of Articles by Members of IFIP WG2.3, Springer New York, New York, NY, pp 42-69, doi: 10.1007/978-1-4612-6315-9_6

Lehman MM (1980) Programs, life cycles, and laws of software evolution. Proceedings of the IEEE 68(9):1060-1076

Li X, Li YF, Xie M, Ng SH (2011) Reliability analysis and optimal version-updating for open source software. Information and Software Technology 53(9):929-936, doi: 10.1016/j.infsof.2011.04.005, studying work practices in Global Software Engineering

Lind RK, Vairavan K (1989) An experimental investigation of software metrics and their relationship to software development effort. IEEE Transanctions on Software Engineering 15(5):649-653, doi: $10.1109 / 32.24715$

Løhre E, Jørgensen M (2016) Numerical anchors and their strong effects on software development effort estimates. Journal of Systems and Software 116:49-56, doi: 10.1016/j.jss.2015.03.015

Mäntylä MV, Lassenius C (2006) Subjective evaluation of software evolvability using code smells: An empirical study. Empirical Software Engineering 11(3):395-431, doi: 10.1007/s10664-006-9002-8

Martinez M, Weimer W, Monperrus M (2014) Do the fix ingredients already exist? an empirical inquiry into the redundancy assumptions of program repair approaches. In: Companion Proceedings of the 36th International Conference on Software Engineering, Association for Computing Machinery, New York, NY, USA, ICSE Companion 2014, pp 492—-495, doi: 10.1145/2591062.2591114

Munaiah N, Kroh S, Cabrey C, Nagappan M (2017) Curating GitHub for engineered software projects. Empirical Software Engineering 22(6):3219-3253, doi: 10.1007/s10664-017-9512-6

Myers EW (1986) An O(ND) difference algorithm and its variations. Algorithmica 1(1-4):251-266, doi: 10.1007/BF01840446

Nguyen HA, Nguyen AT, Nguyen TT, Nguyen TN, Rajan H (2013) A study of repetitiveness of code changes in software evolution. In: 28th IEEE/ACM International Conference on Automated Software Engineering (ASE), pp 180-190

Nguyen HV, Nguyen HA, Nguyen TT, Nguyen AT, Nguyen TN (2012) Detection of embedded code smells in dynamic web applications. In: Proceedings of the 27th IEEE/ACM International Conference on Automated Software Engineering, Association for Computing Machinery, New York, NY, USA, ASE 2012, pp 282-285, doi: 10.1145/2351676.2351724

Nugroho YS, Hata H, Matsumoto K (2020) How different are different diff algorithms in Git? Empirical Software Engineering 25(1), doi: 10.1007/s10664-019-09772-z

Ozment A, Schechter SE (2006) Milk or wine: Does software security improve with age? In: Proceedings of the 15th Conference on USENIX Security Symposium - Volume 15, USENIX Association, Berkeley, CA, USA, USENIX-SS'06

Padgett WJ (2011) Weibull distribution. In: Lovric M (ed) International Encyclopedia of Statistical Science, Springer Berlin Heidelberg, Berlin, Heidelberg, pp 1651-1653, doi: 10.1007/ 978-3-642-04898-2_611

Parnas DL (1994) Software aging. In: Proceedings of the 16th International Conference on Software Engineering, IEEE Computer Society Press, Los Alamitos, CA, USA, ICSE '94, pp 279-287 
Penta MD, Cerulo L, Aversano L (2009) The life and death of statically detected vulnerabilities: An empirical study. Information and Software Technology 51(10):1469 - 1484, doi: https://doi.org/10. 1016/j.infsof.2009.04.013, source Code Analysis and Manipulation, SCAM 2008

Petke J, Harman M, Langdon WB, Weimer W (2014) Using genetic improvement and code transplants to specialise a C++ program to a problem class. In: Nicolau M, Krawiec K, Heywood MI, Castelli M, García-Sánchez P, Merelo JJ, Rivas Santos VM, Sim K (eds) Genetic Programming, Springer Berlin Heidelberg, Berlin, Heidelberg, pp 137-149

Petke J, Haraldsson SO, Harman M, Langdon WB, White DR, Woodward JR (2018) Genetic improvement of software: A comprehensive survey. IEEE Transactions on Evolutionary Computation 22(3):415-432, doi: 10.1109/TEVC.2017.2693219

Qin F, Tucek J, Sundaresan J, Zhou Y (2005) Rx: Treating bugs as allergies-a safe method to survive software failures. In: Proceedings of the 20th ACM Symposium on Operating Systems Principles, ACM, New York, NY, USA, SOSP '05, pp 235-248, doi: 10.1145/1095810.1095833

Robillard MP, Murphy GC (2007) Representing concerns in source code. ACM Transactions on Software Engineering and Methodology 16(1), doi: 10.1145/1189748.1189751

Robles G, Amor JJ, Gonzalez-Barahona JM, Herraiz I (2005) Evolution and growth in large libre software projects. In: Eighth International Workshop on Principles of Software Evolution (IWPSE'05), pp 165-174, doi: 10.1109/IWPSE.2005.17

Robles G, González-Barahona JM, Herraiz I (2005) An empirical approach to software archaeology. In: In Poster Proceedings of the 2005 International Conference on Software Maintenance, ICSM '05, pp $47-50$

Rodríguez D, Sicilia M, García E, Harrison R (2012) Empirical findings on team size and productivity in software development. Journal of Systems and Software 85(3):562-570, doi: 10.1016/j.jss.2011.09.009, novel approaches in the design and implementation of systems/software architecture

Salfner F, Lenk M, Malek M (2010) A survey of online failure prediction methods. ACM Computing Surveys 42(3):10:1-10:42, doi: 10.1145/1670679.1670680

Samoladas I, Angelis L, Stamelos I (2010) Survival analysis on the duration of open source projects. Information and Software Technology 52(9):902-922

Scanniello G (2011) Source code survival with the Kaplan Meier. In: Proceedings of the 27th IEEE International Conference on Software Maintenance, ICSM '11, pp 524-527, doi: 10.1109/ICSM.2011. 6080823

Sentas P, Angelis L, Stamelos I (2008) A statistical framework for analyzing the duration of software projects. Empirical Software Engineering 13(2):147-184

Servant F, Jones JA (2017) Fuzzy fine-grained code-history analysis. In: Proceedings of the 49th International Conference on Software Engineering, IEEE Computer Society Press, Los Alamitos, CA, USA, ICSE '17, doi: 10.1109/ICSE.2017.74

Sharma T, Fragkoulis M, Spinellis D (2016) Does your configuration code smell? In: 2016 IEEE/ACM 13th Working Conference on Mining Software Repositories (MSR), IEEE Computer Society, Los Alamitos, CA, USA, pp 189-200, doi: 10.1109/MSR.2016.028

Shin Y, Meneely A, Williams L, Osborne JA (2010) Evaluating complexity, code churn, and developer activity metrics as indicators of software vulnerabilities. IEEE Transactions on Software Engineering 37(6):772-787

Spinellis D (2016a) How can I obtain with git log a series of patches that can be auto-applied? URL http://stackoverflow.com/q/38839595/20520, archived through WebCite at http: //wWw. webcitation.org/6jyf48Ue7 
Spinellis D (2016b) A repository of Unix history and evolution. Empirical Software Engineering doi: 10.1007/s10664-016-9445-5

Spinellis D (2019) dspinellis/tokenizer: Version 1.1. doi: 10.5281/zenodo.2558420, https://github.com/dspinellis/tokenizer/

Spinellis D, Kotti Z, Mockus A (2020) A dataset for GitHub repository deduplication. In: 17th International Conference on Mining Software Repositories, Association for Computing Machinery, New York, NY, USA, MSR '20, pp 523-527, doi: 10.1145/3379597.3387496

Stamelos I, Angelis L, Oikonomou A, Bleris GL (2002) Code quality analysis in open source software development. Information Systems Journal 12(1):43-60, doi: 10.1046/j.1365-2575.2002.00117.x

Vallée-Rai R, Co P, Gagnon E, Hendren L, Lam P, Sundaresan V (2010) Soot: A java bytecode optimization framework. In: CASCON First Decade High Impact Papers, IBM Corp., Riverton, NJ, USA, CASCON '10, pp 214-224, doi: 10.1145/1925805.1925818

Voinea L, Telea A, van Wijk JJ (2005) CVSscan: Visualization of code evolution. In: Proceedings of the 2005 ACM Symposium on Software Visualization, Association for Computing Machinery, New York, NY, USA, SoftVis ?05, p 47?56, doi: 10.1145/1056018.1056025

White M, Vendome C, Linares-Vásquez M, Poshyvanyk D (2015) Toward deep learning software repositories. In: Proceedings of the 12th Working Conference on Mining Software Repositories, IEEE Press, Piscataway, NJ, USA, MSR '15, pp 334-345, doi: 10.1109/MSR.2015.38

Zhang H (2009) An investigation of the relationships between lines of code and defects. In: Proceedings of the 25th IEEE International Conference on Software Maintenance, ICSM '09, pp 274-283, doi: 10.1109/ICSM.2009.5306304

Zheng X, Zeng D, Li H, Wang F (2008) Analyzing open-source software systems as complex networks. Physica A: Statistical Mechanics and its Applications 387(24):6190-6200

Zimmermann T (2006) Fine-grained processing of CVS archives with APFEL. In: Proceedings of the 2006 OOPSLA Workshop on Eclipse Technology EXchange, Association for Computing Machinery, New York, NY, USA, eclipse '06, pp 16—-20, doi: 10.1145/1188835.1188839

Zimmermann T, Zeller A, Weissgerber P, Diehl S (2005) Mining version histories to guide software changes. IEEE Transactions on Software Engineering 31(6):429-445, doi: 10.1109/TSE.2005.72

Zimmermann T, Kim S, Zeller A, Whitehead EJ Jr (2006) Mining version archives for co-changed lines. In: Proceedings of the 2006 International Workshop on Mining Software Repositories, ACM, New York, NY, USA, MSR ’06, pp 72-75, doi: 10.1145/1137983.1138001 